\title{
The Association of Serum Lipid Levels with Breast Cancer Risks Among Women with Breast Cancer at Felege Hiwot Comprehensive Specialized Hospital, Northwest Ethiopia
}

Breast Cancer: Targets and Therapy

\section{Getinet Kumie \\ Tadele Melak iD \\ Habtamu Wondifraw Baynes \\ University of Gondar, College of Medicine \& Health Sciences, School of Biomedical \& Laboratory Sciences, Department of Clinical Chemistry, Gondar, Ethiopia}

Background: Breast cancer is a malignant condition affecting about 2.1 million women worldwide. Cancer cells have increased the level of cholesterol and facilitate lipid biosynthesis and metabolism. Therefore, there is a need for assessment of serum lipid and its association with breast cancer risks.

Methods: A comparative cross-sectional study was conducted among women with breast cancer $(n=23)$, benign breast lump $(n=68)$ and apparently healthy control $(n=91)$ at Felege Hiwot Comprehensive Specialized Hospital, from January to May, 2020. A convenient sampling technique and an interviewer-administered questionnaires were used to collect data. Five milliliter of fasting blood sample was collected for lipid level analysis using enzymatic colorimetric method. Data entry and analysis were done using Epi Info version 7 and SPSS version 20. One way ANOVA and logistic regression were the tools used for analysis, and a p-value of $<0.05$ was considered as statistically significant.

Results: The mean age of healthy control, benign and malignant breast cancer was 32.51 $\pm 9.44,33.34 \pm 10.664$ and $37.65 \pm 14.345$ years, respectively. The mean serum value of TG among controls, benign and malignant women was $168.67 \pm 41.616,170.1 \pm 59.018$ and 211.7 $\pm 82.924(\mathrm{p}<0.001)$, respectively. The mean serum concentration of HDL-c was significantly different between the three groups $(47.61 \pm 9.122,44.69 \pm 14.479$ and $38.26 \pm 7.442: \mathrm{p}=0.004)$ among controls, benign and malignant, respectively. Low serum HDL-c level was significantly associated with age at parity $(\mathrm{AOR}=3.353 ; 95 \% \mathrm{CI}=1.33,8.436)$, the habit of drinking alcohol $(\mathrm{AOR}=2.125 ; 95 \% \mathrm{CI}=1.065,4.241)$ and $\mathrm{BMI}(\mathrm{AOR}=3.555 ; 95 \% \mathrm{CI}=1.13,11.184)$. In addition, BMI (AOR: 4.54; 95\% CI: 1.45, 14.21) was statistically associated with high LDL-c level.

Conclusion: The overall prevalence of dyslipidemia was high in breast cancer patients. Age at first parity, alcohol consumption and BMI were significantly associated with low serum HDL-c level.

Keywords: breast cancer, lipid profile, women, Ethiopia

\section{Background}

Breast cancer develops from breast cells and tissue from the lining of milk ducts and the lobules that supply the ducts with milk. ${ }^{1,2}$ The precise cause of breast cancer is unknown; but, the female sex hormone, estrogen, is a potential carcinogenic promoter for cellular growth in breast tissues and reproductive organs. ${ }^{3}$ In addition, environmental and lifestyle factors, ${ }^{4,5}$ lack or short duration of

\section{Baynes}

University of Gondar, College of Medicine \& Health Sciences, School of Biomedical \& Laboratory Sciences, Department of Clinical Chemistry, P.O. Box: 196, Gondar Ethiopia

Email Habtamuw97@gmail.com 
breastfeeding, low parity, irregular menstrual period, family history of breast cancer, and inherited mutated genes associated with the onset of breast cancer. ${ }^{6}$

According to the global burden of cancer report of 2018, globally there are about 2.1 million breast cancer cases and responsible for $11.6 \%$ of deaths from cancer causes. $^{7}$ It was estimated that women who survive to the age of 85 will have a 1 in 9 lifetime risk of developing breast cancer. ${ }^{8}$ Breast cancer is becoming public health problem of South America, Africa and Asia due to lack of suitable care, lack of information about breast cancer and lack of timely diagnosis and treatment. ${ }^{9}$ It is estimated that by 2025 over 19.3 million women, with the majority from Sub-Saharan Africa, will be at risk for breast cancer and responsible for 1 in 4 diagnosed cancers and 1 in 5 cancer deaths in women. $^{10}$

Different factors are assumed for breast cancer development. Environmental factors such as exposure to radiation and chemicals, ${ }^{6}$ lifestyle factors such as obesity, physical inactivity, increased dietary fat, use of contraceptives, smoking, diabetes mellitus, hypertension, ${ }^{4,11}$ lack or short duration of breastfeeding, irregular menstrual period, family history of breast cancer, inherited mutated genes trigger the onset of breast cancer are assumed for aggravation of breast cancer. ${ }^{6}$

Changes in concentration of serum lipids in patients with breast cancer will result in an increased production of tumor necrosis factor-alpha (TNF- $\alpha$ ) and inhibition of adipose lipoprotein lipase activity by insulin ${ }^{12}$ these changes will impair the catabolism of very low-density lipoprotein cholesterol (VLDL-c), which is influenced by dietary fat intake, alcohol consumption, body weight, pregnancy, endogenous hormones, smoking and physical inactivity. ${ }^{13}$ Besides this, proliferating cells such as cancer cells have increased requirement of cholesterol and facilitate lipid biosynthesis and metabolism that will finally leads to increased serum cholesterol level in patients with breast cancer. ${ }^{14,15}$ In addition, since cholesterol is transported by low-density lipoprotein cholesterol (LDL-c) and high-density lipoprotein cholesterol (HDL-c), abnormal serum level of LDL-c and HDL-c is associated with breast cancer and its normal serum level is considered as prognostic marker for breast tumor. ${ }^{16}$ Increased level of total cholesterol (TC) and triglyceride (TG) stimulates cell proliferation and induce fibrosarcoma or will lead to the decreased level of sex hormone-binding globulin, which is likely to increase breast cancer risk. ${ }^{2}$ Lipid and lipoprotein abnormalities are in alarming higher prevalence with higher cholesterol, TG, low-density lipoprotein (LDL-c) and lower high-density lipoprotein (HDL-c) especially on postmenopausal women with breast cancer. $^{13}$

Therefore, this study aimed to assess the level of serum lipid and its association with breast cancer risks among breast cancer women, benign breast lump and healthy controls attending at Felege Hiwot Comprehensive Specialized Hospital (FHCSH), Northwest Ethiopia.

\section{Methods and Materials}

\section{Study Area, Study Design and Period}

The study was conducted at FHCSH, North West Ethiopia from January 22-2020 to May 26-2020 using a comparative cross-sectional study. The hospital is one of the public hospitals found in Bahir Dar city located $11^{\circ}$ $36^{\prime} \mathrm{N} 37^{\circ} 23^{\prime} \mathrm{E}$ and an altitude of 1800 meters (5900 feet) above sea level and $565 \mathrm{Km}$ away from the Capital Addis Ababa. ${ }^{17}$

\section{Populations}

A total of 182 (68 benign, 23 benign and 91 controls) participants were included in the study. All participants requested for pathological investigation for breast problem and apparently healthy women who were immediate relatives or patient attendants visiting $\mathrm{FHCSH}$, during the study period were used as source population. Age of participants greater than or equal to 18 years and confirmed as having a benign breast lump or malignant breast cancer through Fine Needle Aspiration Cytology (FNAC) investigation were considered as study populations. In addition, apparently healthy controls who were screened for breast cancer through physical examination, urine human chorionic gonadotrophic hormone (HCG), random blood sugar (RBS) and attending the hospital as patient attendants were included, whereas critically ill patients and those having psychiatric problems, the presence of other malignant tumors other than breast cancer and participants on lipid-lowering therapy were excluded from the study. The sample size was determined by double population proportion formula using the mean and standard deviation (mean $\pm \mathrm{SD}$ ) of serum Low-Density Lipoprotein (LDL). The mean \pm SD of LDL-c taken from the study was 109 $\pm 29 \mathrm{mg} / \mathrm{dl}$ from participants with breast cancer and 98 $\pm 23.6 \mathrm{mg} / \mathrm{dl}$ on apparently healthy controls. $^{18}$ Furthermore, participants were recruited using convenient 
sampling technique until the final sample size was achieved.

\section{Data Collection Procedure and Laboratory Methods}

Socio-demographic and clinical characteristic data were collected using an interviewer-administered questionnaire. Weight was measured using calibrated balance and expressed in kilograms with light cloths and in bare feet. In addition, height was measured using a wall mount ruler, while participant standing straight in bare feet: heels and buttocks, shoulders and back of the head touching the upright position. Blood pressure was measured by qualified nurses using an automated Mercury sphygmomanometer at the midpoint of the left arm.

Five milliliters of venous blood were collected using a serum separator test tube and labeled with participant's identification. The collected blood was left on the test tube rack at room temperature for 30 minutes to clot and centrifuged by using a BT-2000 universal centrifuge adjusted at 3000 revolution per minute (RPM) for 5 minutes to separate serum from whole blood. Serum total Cholesterol (TC), Triglycerides (TG), High-Density Lipoprotein (HDL-c) and LowDensity Lipoprotein (LDL-c) were analyzed by enzymatic colorimetric method using the Mindray BS-200E (Shenzhen Mindray Bio-Medical electronics Co. Ltd, China) Clinical Chemistry auto analyzer following the manufacturer's instruction and standard operating procedures. The analyzer was programmed by downloading the specimen ID numbers and test codes (for TC, TG, HDL-c and direct HDL-c, as appropriate) in machinereadable form. The download file is a database prepared from the electronic sample shipment $\log$. TC, TG and HDL-c were analyzed simultaneously. Abnormal lipid level was assumed when serum $\mathrm{TG} \geq 150 \mathrm{mg} / \mathrm{dl}$, serum TC $\geq 200 \mathrm{mg} / \mathrm{dl}$, serum LDL-c $\geq 130 \mathrm{mg} / \mathrm{dl}$, and/or serum LDL-c less than $40 \mathrm{mg} / \mathrm{dl} .{ }^{19}$ Hypertension was considered when systolic blood pressure $\geq 140 \mathrm{~mm} \mathrm{Hg}$ and/or diastolic blood pressure $\geq 90 \mathrm{~mm} \mathrm{Hg}$. ${ }^{20}$

\section{Data Management and Analysis}

Data were entered and checked for its completeness and cleanness by using Epi Info version 7 before analysis. Then, it was exported, coded and analyzed using SPSS version 20. Normality was checked using a Kolmogorov-
Smirnov test. Descriptive statistics were used to summarize socio-demographic, clinical, and anthropometric measurements related to breast cancer and findings were presented using tables. One way ANOVA was used to compare serum lipid levels between controls, benign breast lump, and malignant breast cancer participants. Binary logistic and multiple logistic regression models were used to assess the association between individual explanatory variables and serum lipid level using 95\% CI and a p-value $<0.05$ was considered as statistically significant.

\section{Results}

\section{Socio-Demographic and Behavioral Characteristics}

A total of 182 participants were included in this study. Of them, 91 (50\%) were controls, 68 (37.36\%) were benign breast lump and $23(12.6 \%)$ were malignant breast cancer patients. The mean ages of controls, benign breast lump, and malignant breast cancer patients were $32.51 \pm 9.44,33.34 \pm 10.664$ and $37.65 \pm 14.345$ years, respectively. Fourteen (15.38\%) of controls, 5 (17.65\%) of benign breast lumps, and $2(17.4 \%)$ of malignant breast cancer cases had systolic blood pressure of $\geq 140 \mathrm{mmHg}$. From FNAC results of benign breast lump, 47(54.4\%) were breast fibrinoma and the remaining were benign breast lesions. In addition, from FNAC results of malignant breast cancer, 19(65.2\%) were ductal carcinoma and the remaining were lobular carcinoma (Table 1).

\section{Comparison of Serum Lipid Level of Study Participants}

The mean value of TG among controls, benign and malignant women were significantly different $(168.67 \pm 41.616$, $170.1 \pm 59.018$ and $211.7 \pm 82.924 ; \quad \mathrm{p}<0.001) \quad$ ANOVA results, respectively. In addition, the mean serum concentrations of HDL-c were significantly different between the three groups $(47.61 \pm 9.122,44.69 \pm 14.479$ and 38.26 \pm 7.442 : $\mathrm{p}=0.004)$ among controls, benigns and malignants, respectively (Table 2 ). The overall prevalence of dyslipidemia was $75.27 \%$; and $64.83 \%, 83.82 \%, 91.3 \%$ among controls, benign breast lump and malignant breast tumor, respectively. Moreover, the prevalence of high TG levels among controls, benign breast lump and malignant breast cancer were $46.15 \%, 54.4 \%$ and 69.56 , respectively. The prevalence of abnormal values of TC was statististicaly 
Table I Sociodemographic, Behavioral and Anthropometric Characteristics of Women with Breast Cancer, Benign Breast Lump and Healthy Controls at Felege Hiwot Comprehensive Specialized Hospital, Northwest Ethiopia, 2020 (N=182)

\begin{tabular}{|c|c|c|c|c|}
\hline \multicolumn{2}{|l|}{ Variables } & \multirow{2}{*}{$\begin{array}{l}\text { Malignant }(n=23) \\
\text { Number (\%) }\end{array}$} & \multirow{2}{*}{$\begin{array}{l}\text { Benign }(\mathrm{n}=68) \\
\text { Number }(\%)\end{array}$} & \multirow{2}{*}{$\begin{array}{l}\text { Control }(n=91) \\
\text { Number }(\%)\end{array}$} \\
\hline & & & & \\
\hline \multirow[t]{4}{*}{ Age in year } & $18-24$ & $6(26.09 \%)$ & $2 \mathrm{I}(30.88 \%)$ & $24(26.37 \%)$ \\
\hline & $24-34$ & $5(21.74 \%)$ & $18(26.47 \%)$ & 37 (40.66\%) \\
\hline & $34-45$ & 7 (30.44\%) & 22 (32.35\%) & $22(24.17 \%)$ \\
\hline & $\geq 45$ & 5 (21.73\%) & $7(10.3 \%)$ & $8(8.8 \%)$ \\
\hline \multirow[t]{2}{*}{ Residence } & Urban & II (47.82\%) & 38 (55.88\%) & 49 (53.8\%) \\
\hline & Rural & $12(52.17 \%)$ & $30(44.12 \%)$ & $42(46.2 \%)$ \\
\hline \multirow[t]{3}{*}{ Marital status } & Single & $4(17.4 \%)$ & $17(25 \%)$ & $32(35.16 \%)$ \\
\hline & Married & 19 (82.6\%) & $43(63.23 \%)$ & $5 \mathrm{I}(56.04 \%)$ \\
\hline & Divorced/widowed & 0 & 8 (II.77\%) & $8(8.8 \%)$ \\
\hline \multirow[t]{4}{*}{ Educational status } & No education & 13 (56.52\%) & 29 (42.64\%) & $34(37.36 \%)$ \\
\hline & Elementary school & $5(21.74 \%)$ & $22(32.35 \%)$ & 30 (32.97\%) \\
\hline & High school & 5 (21.74\%) & 9 (I3.23\%) & $24(26.37 \%)$ \\
\hline & College/university & 0 & 8 (II.78\%) & $3(3.3 \%)$ \\
\hline \multirow[t]{2}{*}{ Alcohol drinking habit } & Yes & $10(43.48 \%)$ & $25(36.76 \%)$ & 45 (49.45\%) \\
\hline & No & $13(56.52 \%)$ & 43 (63.24\%) & 46 (50.55\%) \\
\hline \multirow[t]{2}{*}{ Contraceptive use } & Yes & II (47.82\%) & 40 (58.82\%) & 37 (40.66\%) \\
\hline & No & $12(52.18 \%)$ & $28(41.18 \%)$ & 54 (59.34\%) \\
\hline \multirow[t]{2}{*}{ Abortion history } & Yes & $4(17.4 \%)$ & $5(7.36 \%)$ & II (I2.08\%) \\
\hline & No & $19(62.6 \%)$ & $63(92.64 \%)$ & 80 (87.92\%) \\
\hline \multirow[t]{3}{*}{ Age at parity (in year) } & $<22$ & $12(52.17 \%)$ & 27 (39.7\%) & 30 (32.97\%) \\
\hline & $\geq 22$ & $5(21.74 \%)$ & $13(19.11 \%)$ & 17 (18.68\%) \\
\hline & Nulliparous & $6(26.09 \%)$ & $28(4 \mid .19 \%)$ & 44 (48.35\%) \\
\hline \multirow[t]{2}{*}{ Age menarche (in year) } & $<16$ & $10(43.48 \%)$ & $30(44.11 \%)$ & 55 (60.44\%) \\
\hline & $\geq 16$ & $13(56.52 \%)$ & $38(55.89 \%)$ & $36(39.56 \%)$ \\
\hline \multirow[t]{2}{*}{ Menopause status } & Pre-menopause & 18 (78.26\%) & $58(85.3 \%)$ & $82(90.1 \%)$ \\
\hline & Post-menopause & $5(21.74 \%)$ & $10(14.7 \%)$ & 9 (9.9\%) \\
\hline \multirow[t]{2}{*}{ Menstruation } & Irregular & $9(39.13 \%)$ & $24(35.3 \%)$ & 29 (31.9\%) \\
\hline & Regular & 14 (60.87\%) & $44(64.7 \%)$ & $62(68.1 \%)$ \\
\hline \multirow[t]{2}{*}{ Regular exercise } & Yes & $2(8.7)$ & $7(10.3 \%)$ & $20(21.98 \%)$ \\
\hline & No & $21(91.3 \%)$ & $61(89.7 \%)$ & $71(78.02 \%)$ \\
\hline \multirow[t]{3}{*}{ BMI $\left(\mathrm{kg} / \mathrm{m}^{2}\right)$} & $<18.5$ & $I(4.36 \%)$ & $5(0.35 \%)$ & I (I.I\%) \\
\hline & $18.5-24.9$ & $19(82.6 \%)$ & $56(82.35 \%)$ & $84(92.3 \%)$ \\
\hline & $\geq 25$ & $3(13.04 \%)$ & 7 (10.3\%) & $6(6.6 \%)$ \\
\hline \multirow[t]{2}{*}{$\mathrm{SBP}(\mathrm{mmHg})$} & $<140$ & 19 (82.6\%) & $56(82.35 \%)$ & 77 (84.62\%) \\
\hline & $\geq 140$ & $2(17.4 \%)$ & $5(17.65 \%)$ & $14(15.38 \%)$ \\
\hline \multirow[t]{2}{*}{$\mathrm{DBP}(\mathrm{mmHg})$} & $<90$ & 21 (91.3\%) & $64(94.1 \%)$ & $82(90.1 \%)$ \\
\hline & $\geq 90$ & $2(8.7 \%)$ & $4(5.9 \%)$ & $9(9.9 \%)$ \\
\hline
\end{tabular}

different between controls, benign and malignant (3.3\%, $11.76 \%$ and $17.39 \%$ : $p=0.037$ ) respectively. On the other hand, the abnormally low values of HDL-c were significantly different between controls, benign and malignant $(20.88 \%, 42.64 \%$ and $60.87 \%: \mathrm{p}<0.001)$ respectively (Table 3). 
Table 2 One Way ANOVA Test for Serum Lipid Level Between Breast Cancer, Benign Breast Lump and Apparently Healthy Controls Women at Felege Hiwot Comprehensive Specialized Hospital, Northwest Ethiopia, 2020 ( $n=182)$

\begin{tabular}{|c|c|c|c|c|}
\hline Lipid Components & Control (N=9l) Mean士SD & Benign $(\mathrm{N}=68)$ Mean $\pm S D$ & Malignant ( $\mathrm{N}=23$ ) Mean $\pm S D$ & P-value \\
\hline Serum TC (mg/dl) & $141.37 \pm 33.206$ & $|64.76 \pm 42.36|$ & $174.43 \pm 26.015$ & 0.607 \\
\hline Serum TG (mg/dl) & $168.67 \pm 41.616$ & $170.1 \pm 59.018$ & $211.7 \pm 82.924$ & $<0.001$ \\
\hline Serum LDL-c (mg/dl) & $90.76 \pm 38.065$ & $89.24 \pm 30.291$ & $98.52 \pm 39.812$ & 0.626 \\
\hline Serum HDL-c $(m g / d l)$ & $47.61 \pm 9.122$ & $44.69 \pm 14.479$ & $38.26 \pm 7.442$ & 0.004 \\
\hline
\end{tabular}

Abbreviations: TC, total cholesterol; TG, triglycerides; LDL-c, low-density lipoprotein cholesterol; HDL-c, high-density lipoprotein cholesterol.

Table 3 Prevalence of Dyslipidemia and Components Abnormality Among Controls, Benign Breast Lump and Malignant Breast Tumor at Felege Hiwot Comprehensive Specialized Hospital, Northwest Ethiopia, $2020(n=182)$

\begin{tabular}{|c|c|c|c|c|c|}
\hline \multicolumn{2}{|c|}{ Components of Lipid Level } & \multirow{3}{*}{$\begin{array}{l}\text { Control (91) n (\%) } \\
88(96.7) \\
3(3.3)\end{array}$} & \multirow{3}{*}{$\begin{array}{l}\text { Benign (68) n(\%) } \\
60(88.24) \\
8(11.76)\end{array}$} & \multirow{3}{*}{$\begin{array}{l}\text { Malignant (23) n (\%) } \\
19(82.61) \\
4(17.39)\end{array}$} & \multirow{3}{*}{$\begin{array}{l}\text { P-value } \\
0.037\end{array}$} \\
\hline $\mathrm{TC}(\mathrm{mg} / \mathrm{dl})$ & $<200$ & & & & \\
\hline & $\geq 200$ & & & & \\
\hline \multirow[t]{2}{*}{ TG (mg/dl) } & $<150$ & 49 (53.85) & $31(45.6)$ & 7 (30.44) & \\
\hline & $\geq 150$ & $42(46.15)$ & $37(54.4)$ & $16(69.56)$ & 0.120 \\
\hline \multirow[t]{2}{*}{ LDL-c (mg/dl) } & $<130$ & 80 (87.92) & $60(88.24)$ & 17 (73.92) & \\
\hline & $\geq 130$ & II (I2.08\%) & $8(1 \mathrm{I} .76)$ & $6(26.08)$ & 0.183 \\
\hline \multirow[t]{2}{*}{ HDL-c (mg/dl) } & $<40$ & $19(20.88)$ & $29(42.64)$ & $14(60.87)$ & $<0.001$ \\
\hline & $\geq 40$ & $72(79.12)$ & $39(57.36)$ & $9(39.13)$ & \\
\hline
\end{tabular}

\section{Association of Breast Cancer Risk Factors with Serum Lipid Levels}

The association of socio-demographic and behavioral risk association with serum lipid level were evaluated using binary and multivariable logistic regressions. The binary logistic analysis revealed that age at first parity, habit of drinking alcohol and BMI were significantly associated with low serum HDL-c level $[(\mathrm{COR}=3.05 ; 95 \% \mathrm{CI}=$ $1.31,7.08),(\mathrm{COR}=2.1 ; 95 \% \mathrm{CI}=1.106,3.99), \mathrm{COR}=$ $3.65 ; 95 \% \mathrm{CI}=1.261,10.588)]$, respectively (Table 4 ). Variables having $\mathrm{p}<0.2$ in the binary logistic analysis were further analyzed by multiple logistic regression. Based on the analysis age of parity (AOR: $3.353 ; 95 \%$ CI: 1.33 , 8.436), habit of drinking alcohol (AOR: $2.125 ; 95 \% \mathrm{CI}$ : $1.065,4.241$ ), breast cancer status (benign breast lump) (AOR: 5.32; 95\% CI: 1.95, 14.53) and BMI (AOR: 3.55; 95\% CI: $1.13,11.18)$ were significantly associated with low serum HDL-c level. In addition, BMI (AOR: 4.54; $95 \%$ CI: $1.45,14.21)$ was statistically associated for high TG level (Table 5).

\section{Discussion}

Lipids are water-insoluble molecules that are important in maintaining the structural and functional integrity of membranes, cellular signaling, cell transformation, cancer progression and metastasis. ${ }^{21}$ Studies indicated that lipidlowering medications had improved outcomes on breast cancer patients on hormonal therapy. ${ }^{22}$ Thus, for effective breast cancer treatment and for breast cancer comorbidities reduction, evaluation of serum lipid levels is an important parameter.

The overall prevalence of dyslipidemia found in this study was $75.27 \%$. Besides this, $64.83 \%$ controls, $83.82 \%$ benign breast lump, $91.3 \%$ malignant breast cancer develop dyslipidemia, which was higher than the study conducted in Italy $^{23}$ and lower than from Brazil. $^{5}$ In contrary to this study, the prevalence of dyslipidemia was lower among malignant breast cancer as compared to controls in China. This can be due to differences in study participants (breast cancer patients on chemotherapy for the study in China) and it can be due to the effect of chemotherapy on breast cancer cells and serum lipid profile level. ${ }^{24}$ This study showed the prevalence of hypertriglyceridemia concentration among the whole study participants, controls, benign breast lump and malignant breast cancers as $52.2 \%, 46.15 \%$, $54.4 \%$ and $69.56 \%$, respectively. Similarly, high level of LDL-c was observed in $13.73 \%$ of the whole study participants, $12.08 \%$ of controls, $11.76 \%$ of benign 
Table 4 Binary Logistic Regression of Anthropometric Measurement and Behavioral Variables with Serum Lipid Level Among Controls, Benign Breast Lump and Malignant Breast Tumor Women at Felege Hiwot Comprehensive Specialized Hospital, Northwest Ethiopia, 2020 ( $\mathrm{n}=182$ )

\begin{tabular}{|c|c|c|c|c|c|}
\hline Variables & & $\mathrm{TC} \geq 200 \mathrm{mg} / \mathrm{dl}$ & TG $\geq 150 \mathrm{mg} / \mathrm{dl}$ & LDL-c $\geq 130 \mathrm{mg} / \mathrm{dl}$ & HDL-c $<40 \mathrm{mg} / \mathrm{dl}$ \\
\hline Post-menopausal & $\begin{array}{l}\text { P- value } \\
\text { COR }(\mathrm{Cl})\end{array}$ & $\begin{array}{l}0.447 \\
0.45(0.05,3.56)\end{array}$ & $\begin{array}{l}0.817 \\
0.90(0.38,2.13)\end{array}$ & $\begin{array}{l}0.655 \\
0.1 .30(0.41,4.19)\end{array}$ & $\begin{array}{l}0.703 \\
0.84(0.34,2.05)\end{array}$ \\
\hline $\mathrm{BMI} \geq 25$ & $\begin{array}{l}\text { P-value } \\
\text { COR(Cl) }\end{array}$ & $\begin{array}{l}0.018^{* *} \\
4.69(1.30,17.00)\end{array}$ & $\begin{array}{l}0.224^{*} \\
0.52(0.18,1.49)\end{array}$ & $\begin{array}{l}0.007^{* *} \\
4.64(1.52,14.22)\end{array}$ & $\begin{array}{l}0.017^{* *} \\
3.65(1.26,10.59)\end{array}$ \\
\hline $\mathrm{DBP} \geq 90$ & $\begin{array}{l}\text { P-value } \\
\text { COR(Cl) }\end{array}$ & $\begin{array}{l}0.779 \\
1.36(0.16,0.84)\end{array}$ & $\begin{array}{l}0.026 * * \\
4.44(1.195,16.49)\end{array}$ & $\begin{array}{l}0.95 \\
1.05(0.22,5.00)\end{array}$ & $\begin{array}{l}0.472 \\
0.67(0.22,2.02)\end{array}$ \\
\hline Age at menarche $<16$ & $\begin{array}{l}\text { P-value } \\
\text { COR (Cl) }\end{array}$ & $\begin{array}{l}0.328 \\
1.71(0.58,5.02)\end{array}$ & $\begin{array}{l}0.287 \\
1.37(0.77,2.46)\end{array}$ & $\begin{array}{l}0.983 \\
1.01(0.43,2.35)\end{array}$ & $\begin{array}{l}0.91 \\
1.04(0.56,1.91)\end{array}$ \\
\hline Age at parity $<22$ years & $\begin{array}{l}\text { P-value } \\
\text { COR(Cl) }\end{array}$ & $\begin{array}{l}0.454 \\
0.54(0.11,2.73)\end{array}$ & $\begin{array}{l}0.00 I^{* *} \\
4.09(1.72,9.75)\end{array}$ & $\begin{array}{l}0.977 \\
0.98(0.31,3.14)\end{array}$ & $\begin{array}{l}0.0 I^{* *} \\
3.05(I .31,7.08)\end{array}$ \\
\hline Alcohol drinkers & $\begin{array}{l}\text { P-value } \\
\text { COR(Cl) }\end{array}$ & $\begin{array}{l}0.447 \\
I .5 I(0.52,4.35)\end{array}$ & $\begin{array}{l}0.333 \\
I .34(0.743,2.4 I)\end{array}$ & $\begin{array}{l}0.996 \\
1.00(0.43,2.35)\end{array}$ & $\begin{array}{l}0.023^{* *} \\
2.1(1.11,3.99)\end{array}$ \\
\hline Having abortion & $\begin{array}{l}\text { P-value } \\
\text { COR(Cl) }\end{array}$ & $\begin{array}{l}0.762 \\
1.27(0.27,6.10)\end{array}$ & $\begin{array}{l}0.229 * \\
1.81(0.69,4.77)\end{array}$ & $\begin{array}{l}0.862 \\
1.12(0.30,4.15)\end{array}$ & $\begin{array}{l}0.685 \\
1.23(0.45,3.38)\end{array}$ \\
\hline Lack of physical activity & $\begin{array}{l}\text { P-value } \\
\text { COR(Cl) }\end{array}$ & $\begin{array}{l}0.654 \\
0.74(0.19,2.79)\end{array}$ & $\begin{array}{l}0.338 \\
1.42(0.64,3.16)\end{array}$ & $\begin{array}{l}0.992 \\
0.99(0.31,3.14)\end{array}$ & $\begin{array}{l}0.104 * \\
0.45(0.17,1.17)\end{array}$ \\
\hline Using contraceptive & $\begin{array}{l}\text { P-value } \\
\text { COR(Cl) }\end{array}$ & $\begin{array}{l}0.687 \\
1.24(0.43,3.58)\end{array}$ & $\begin{array}{l}0.782 \\
0.92(0.52,1.65)\end{array}$ & $\begin{array}{l}0.97 \\
0.98(0.42,2.29)\end{array}$ & $\begin{array}{l}0.527 \\
0.82(0.44,1.52)\end{array}$ \\
\hline
\end{tabular}

Notes: *Independent variable eligible for multiple logistic regression $(p \leq 0.25)$, **Statistical significant $(p<0.05)$.

Abbreviations: $\mathrm{COR}$, crude odds ratio; $\mathrm{Cl}, 95 \%$ confidence interval.

Table 5 Multiple Logistic Regression of Anthropometric and Behavioral Variables with Serum Lipid Level Among Controls, Benign Breast Lump and Malignant Breast Tumor Women at Felege Hiwot Comprehensive Specialized Hospital, Northwest Ethiopia, 2020 ( $\mathrm{n}=182$ )

\begin{tabular}{|c|c|c|c|c|c|}
\hline Variables & & $\mathrm{TC} \geq 200 \mathrm{mg} / \mathrm{dl}$ & $T G \geq 150 \mathrm{mg} / \mathrm{dl}$ & LDL-c $\geq 130 \mathrm{mg} / \mathrm{dl}$ & HDL-c $<40 \mathrm{mg} / \mathrm{dl}$ \\
\hline $\mathrm{BMI} \geq 25$ & $\begin{array}{l}\text { P-value } \\
\text { AOR(Cl) }\end{array}$ & $\begin{array}{l}0.31 \\
2.73(0.39,18.90)\end{array}$ & $\begin{array}{l}0.264 \\
0.52(0.16,1.64)\end{array}$ & $\begin{array}{l}0.009 * \\
4.54(|.45,| 4.2 \mid)\end{array}$ & $\begin{array}{l}0.03^{*} \\
3.55(1.13,11.18)\end{array}$ \\
\hline $\mathrm{DBP} \geq 90$ & $\begin{array}{l}\text { P-value } \\
\text { AOR(Cl) }\end{array}$ & $\begin{array}{l}- \\
-\end{array}$ & $\begin{array}{l}0.058 \\
3.742(0.96,14.63)\end{array}$ & - & - \\
\hline Lack of physical activity & $\begin{array}{l}\text { P-value } \\
\text { AOR }(\mathrm{Cl})\end{array}$ & - & - & - & $\begin{array}{l}0.339 \\
0.61(0.22,1.68)\end{array}$ \\
\hline Age at parity $<22$ & $\begin{array}{l}\text { P-value } \\
\text { AOR(Cl) }\end{array}$ & $\begin{array}{l}0.483 \\
0.55(0.10,2.93)\end{array}$ & - & - & $\begin{array}{l}0.01 * \\
3.35(1.33,8.44)\end{array}$ \\
\hline Alcohol drinkers & $\begin{array}{l}\text { P-value } \\
\text { AOR }(\mathrm{Cl})\end{array}$ & - & $\begin{array}{l}- \\
-\end{array}$ & - & $\begin{array}{l}0.033^{*} \\
2.13(1.07,4.24)\end{array}$ \\
\hline Having abortion & $\begin{array}{l}\text { P-value } \\
\text { AOR }(\mathrm{Cl})\end{array}$ & - & $\begin{array}{l}0.233 \\
1.83(0.68,4.68)\end{array}$ & - & - \\
\hline
\end{tabular}

Notes: Independent variables with $p$-value $>0.25$ under binary logistic regression. $*$ Statistical significant association $(p \leq 0.05)$.

Abbreviations: AOR, adjusted odds ratio; $\mathrm{Cl}$, confidence interval.

breast lump and $26.08 \%$ of the malignant breast cancers. Thus, the results of this study were lower than the study done in Brazil. ${ }^{5}$ The difference between the two studies might be due to age, family history, smoking condition, diabetes, genetic and socioeconomic difference of study participants. 
Similarly, there was significantly lower (p-value $=0.008$ ) mean serum levels of HDL-c observed among women with malignant breast cancer $(38.26 \pm 7.44)$ as compared to benign breast lump $(44.69 \pm 14.48)$ and controls $(47.61 \pm 9.12),(p=0.004)$. This finding contradicts from the studies in Taiwan ${ }^{25}$ and agrees with studies from Iraq. ${ }^{26}$ The reason for decreased mean serum HDL-c concentration on malignant breast cancer can be due to abundant expression of scavenger receptor class B type I in human breast tissue which serves as an HDL receptor and mediate its cholesterol uptake. ${ }^{16}$ This study showed significantly $(\mathrm{p}<0.001)$ higher mean serum levels of TG among malignant breast cancers $(211.7 \pm 82.924)$ as compared to benign breast lump $(170.1 \pm 59.018)$ and controls (168.67 \pm 41.62$)$ which concords with the study from Nepalese. ${ }^{21}$ On contrary, a study in Taiwan showed no significant difference in mean serum TG level between apparently health and women with malignant breast tumor. $^{25}$

The finding in this study indicated, higher mean serum level of TC among malignant breast tumor participants as compared to benign breast lump control groups. Similar studies conducted in Iraq and North and central India meet with our study findings. ${ }^{26-28}$ The reason for high serum TC in women with malignant breast tumor can be due to high cholesterol metabolites, 27-hydroxycholesterol, that function as estrogen and increase proliferation of estrogen receptor-positive breast cancer cells. ${ }^{29}$ On contrary, studies conducted in Morocco and India showed no statistically significant difference in mean serum TC level between malignant breast tumor and control groups. ${ }^{30,31}$

This study tried to assess the association between serum lipid levels with breast cancer status and risks of breast cancer. The low serum HDL-c levels were 3.35 times higher among women who gave first birth at the age of $<22$ years as compared to women who gave first birth $\geq 22$ years (CI: $1.33,8.44)$. Similarly, the level of HDL-c among malignant breast cancer was 2.43 times lower than controls (CI: 1.01, 5.83) which concords with a study conducted in Taiwan. ${ }^{25}$ This study came up with HDL-c and LDL-c were significantly associated with BMI. This finding was in line with the study done in London that showed as the BMI increased, the HDL-c concentration also decreased significantly and LDL-c increased. ${ }^{32}$ In addition, the current study showed that low serum HDL-c level was 3.56 times higher among women having BMI $\geq 25 \mathrm{~kg} / \mathrm{m}^{2}$ as compared to BMI $<25 \mathrm{~kg} / \mathrm{m}^{2}$ participants (CI: 1.13, 11.18). This indicates that HDL-c and LDL-c have a potential to be used as a marker and obesity is a candidate for breast cancer development.

The current study showed that high serum TC level was 2.39 times higher among benign breast lump than control groups (CI: 0.407, 14.08). Besides, high serum TG level was 4.54 times higher among women having BMI $\geq 25 \mathrm{~kg} / \mathrm{m}^{2}$ as compared to women having BMI $<25 \mathrm{~kg} / \mathrm{m}^{2}$ (CI: $1.45,14.21$ ) which concords with finding in Morocco that reported a significant association between serum TG level and BMI. ${ }^{31}$ On the other hand recent studies reported that obesity as a common risk for breast cancer and dyslipidemia. ${ }^{18,33,34}$ This could be due to adiposity-related secretion of estrogen, increases in circulating insulin and insulin-like growth factors, local production of adipokines and leptin that could stimulate the breast cell growth. ${ }^{35}$ In addition, obesity decreases the production of sex hormone-binding globulin which in turn results foran increased biologically active unbound form of estrone and estradiol which promotes cell growth and metastatic potential in breast tumor.

This study demonstrated that menopausal status was not significantly associated with serum lipid level which contradicts from studies conducted in India that reported TC, TG, and LDL-c were significantly higher on postmenopausal women than Pre-menopausal women. ${ }^{36}$ A recent study conducted in Cameroon revealed that significantly high TC, LDL-c, TG, and lower HDL-c were observed among obese women participants as compared to normal weight. ${ }^{36}$ This study showed no significant association between serum lipid level and breast cancer risks like the habit of smoking, low physical activity, early menarche, history of abortion, contraceptive use. It contradicts from studies in Paris that reported a significant association between higher serum TG and menopausal status and India which reported significant association between high serum TC, TG and LDL-c and low HDL-c level and BMI. ${ }^{37,38}$ This discrepancy among findings might be due to variation in race, culture, socio-economic status, health status, lifestyle, family history, lack of exercise, smoking, diabetes and age.

\section{Strength, and Limitation of the Study Strengths}

The study involved apparently healthy controls, benign breast lump, and malignant breast tumor that clearly showed the difference in serum lipid levels and the associated risk factors across the groups. 


\section{Limitation}

Since it was a cross-sectional study, it does not show the causal relationship between lipid level abnormality and breast cancer status.

\section{Conclusion and Recommendation}

The Overall prevalence of dyslipidemia was high on breast cancer patients. The mean value of components of lipid levels was higher in breast cancer patients than in controls. There was significantly lower mean serum HDL-c level among malignant breast cancer than benign breast lump and the controls. There was significantly high serum TC on malignant breast cancer than benign breast lump and controls. Low parity, alcohol drinking habit and high BMI were significantly associated with low serum HDL-c levels. On the other hand, high BMI was significantly associated with LDLc level. This indicates that HDL-c and LDL-c have a potential to be used as a marker of diagnostic and prognostic potential; and obesity is an indicator of a candidate for breast cancer development. Therefore, breast cancer patients should monitor their lipid levels to reduce comorbidities and adverse breast cancer outcomes. Further longitudinal studies have to be done on serum lipid level and its association with breast cancer stages and molecular breast cancer subtypes using case-control study design with an improved sample size to assess the causal relationship.

\section{Abbreviations}

AOR, adjusted odds ratio; BMI, body mass index; CI, confidence interval; COR, crude odds ratio; FNAC, fine needle aspiration cytology; FHCSH, Felege Hiwot Comprehensive Specialized Hospital; HCG, human chorionic gonadotrophic hormone; HDL-c, high-density lipoprotein cholesterol; LDL-c, low-density lipoprotein cholesterol; $\mathrm{Mg} / \mathrm{dl}$, milligram per deciliter; $\mathrm{mm} \mathrm{Hg}$, millimeter mercury; SPSS, Statistical Package for Social Science; TC, total cholesterol; TG, triglycerides.

\section{Data Sharing Statement}

All data underlying the findings described in the manuscript are fully available without restriction.

\section{Ethics Approval and Consent to Participate}

The study was conducted after ethical approval was obtained from the Research and Ethical Review Committee of School of Biomedical and Laboratory
Science, College of Medicine and Health Sciences, University of Gondar. Letter of permission was obtained from the Clinical Director of FHCSH. Written informed consent was obtained from study participants before data collection and all the information obtained were handled confidentially. Participants with pathological results were linked to the concerned physician for further investigation, and treatment. Furthermore, this study was conducted in accordance with the Declaration of Helsinki.

\section{Acknowledgment}

We would like to thank the participants of the study and data collectors.

\section{Author Contributions}

All authors (GK, TM and HWB) made substantial contributions to conception and design, acquisition of data, or analysis and interpretation of data; took part in drafting the article or revising it critically for important intellectual content; agreed to submit to the current journal; gave final approval of the version to be published; and agree to be accountable for all aspects of the work.

\section{Funding}

There was no funding obtained for publication.

\section{Disclosure}

The authors report no conflicts of interest for this work.

\section{References}

1. Denton M. Breast Cancer: Risks, Detection, and Treatment. Greenhaven Publishing LLC; 2017.

2. Calhoun KE, Anderson BO. Lobular Carcinoma in situ of the Breast. The Breast. Elsevier; 2018:553-61. e2.

3. Makama M, Drukker C, Rutgers ET, et al. An association study of established breast cancer reproductive and lifestyle risk factors with tumour subtype defined by the prognostic 70-gene expression signature (MammaPrint ${ }^{\circledR}$ ). Eur J Cancer. 2017;75:5-13. doi:10.1016/j. ejca.2016.12.024

4. Yoo KY, Kang D, Park SK, et al. Epidemiology of breast cancer in Korea: occurrence, high-risk groups, and prevention. J Korean Med Sci. 2002;17(1):1. doi:10.3346/jkms.2002.17.1.1

5. Sousa-e-Silva É, Conde DM, Costa-Paiva L, Martinez EZ, Pinto-Neto AM. Cardiovascular risk in middle-aged breast cancer survivors: a comparison between two risk models. Rev Bras Ginecol Obstet. 2014;36(4):157-162. doi:10.1590/S0100-720320140050.0002

6. Paley PJ. Screening for the major malignancies affecting women: current guidelines. Am J Obstet Gynecol. 2001;184(5):1021-1030. doi:10.1067/mob.2001.111714

7. Bray F, Ferlay J, Soerjomataram I, Siegel RL, Torre LA, Jemal A. Global cancer statistics 2018: GLOBOCAN estimates of incidence and mortality worldwide for 36 cancers in 185 countries. CA Cancer J Clin. 2018;68(6):394-424. 
8. Singletary SE. Rating the risk factors for breast cancer. Ann Surg. 2003;237(4):474. doi:10.1097/01.SLA.0000059969.64262.87

9. Rahimzadeh M, Baghestani AR, Gohari MR, Pourhoseingholi MA. Estimation of the cure rate in Iranian breast cancer patients. Asian Pac J Cancer Prev. 2014;15(12):4839-4842. doi:10.7314/APJCP.20 14.15.12.4839

10. Ferlay J, Shin HR, Bray F, Forman D, Mathers C, Parkin DM. Estimates of worldwide burden of cancer in 2008: GLOBOCAN 2008. Int J Cancer. 2010;127(12):2893-2917. doi:10.1002/ijc.25516

11. Conde D, Costa-Paiva L, Martinez E, Pinto-Neto A. Cardiovascular risk in middle-aged breast cancer survivors: a comparison between two risk models [risco Cardiovascular Em Mulheres De Meia-idade Com Câncer De Mama: uma Comparação Entre Dois Modelos De Risco]. Rev Bras Ginecol Obstet. 2014.

12. Knapp ML, Al-Sheibani S, Riches PG. Alterations of serum lipids in breast cancer: effects of disease activity, treatment, and hormonal factors. Clin Chem. 1991;37(12):2093-2101. doi:10.1093/clinchem/ 37.12.2093

13. Mariam MBB, Devi D. The prevalence of dyslipidemia among postmenopausal women in Bangalore (urban area). Int $J$ Emerg Trends Sci Technol. 2016;3(3):3718-3724.

14. Lingwood D, Simons K. Lipid rafts as a membrane-organizing principle. Science. 2010;327(5961):46-50. doi:10.1126/science.1174621

15. Menendez JA, Lupu R. Fatty acid synthase and the lipogenic phenotype in cancer pathogenesis. Nat Rev Cancer. 2007;7(10):763-777. doi: $10.1038 / \mathrm{nrc} 2222$

16. Cedó L, Reddy ST, Mato E, Blanco-Vaca F, Escolà-Gil JC. HDL and LDL: potential new players in breast cancer development. $J$ Clin Med. 2019;8(6):853. doi:10.3390/jcm8060853

17. Dagmawe N In depth investigation of relationship between index property and swelling characteristic of expansive soil in Bahir Dar [Unpublished MSC Thesis]. Eurohazcon Collaboration Addis Ababa University. 2007.

18. Peela J, Jarari A, El Saiety S, El Busaifi S, Srikumar S. The relationship between serum lipids and breast cancer in Libya. Biochem Anal Biochem. 2012;1(6):1-3. doi:10.4172/2161-1009.1000117

19. Khader YS, Batieha A, El-Khateeb M, Al Omari M, Ajlouni K. Prevalence of dyslipidemia and its associated factors among jordanian adults. J Clin Lipidol. 2010;4(1):53-58. doi:10.1016/j.jacl.2009.12.004

20. Organization WH. Waist Circumference and Waist-Hip Ratio: Report of a WHO Expert Consultation. Vol. 8-11. Geneva: December, 2008:2011.

21. Pandeya DR, Rajbhandari A, Nepal M, et al. Comparative study of serum lipid profiles in nepalese cancer patients attending a Tertiary Care Hospital. Asian Pac J Cancer Prev. 2018;19(2):491.

22. Coates A, Ahern T, Borgquist S, Colleoni M, Debled M, Ejlertsen B, editors. Cholesterol, cholesterol-lowering medication use, and breast cancer outcome in the BIG 1-98 study. Am Soc Clin Oncol. 2017;35 (11):1179-1188

23. Sinagra D, Amato C, Scarpitta A, et al. Metabolic syndrome and breast cancer risk. Eur Rev Med Pharmacol Sci. 2002;6:55-59.
24. Li X, Liu Z-L, Wu Y-T, et al. Status of lipid and lipoprotein in female breast cancer patients at initial diagnosis and during chemotherapy. Lipids Health Dis. 2018;17(1):91. doi:10.1186/s12944-018-0745-1

25. Chang S-J, Hou M-F, Tsai S-M, et al. The association between lipid profiles and breast cancer among Taiwanese women. Clin Chem Lab Med. 2007;45(9):1219-1223. doi:10.1515/CCLM.2007.263

26. Abd AA. Assessment of lipid profile parameters in women with benign and malignant breast tumor. Muthanna Med J. 2019;6(1):13-25.

27. Kachhawa P, Kachhawa K, Agrawal D, Sinha V, Sarkar PD, Kumar S. Association of dyslipidemia, increased insulin resistance, and serum CA 15-3 with increased risk of breast cancer in urban areas of North and Central India. J Midlife Health. 2018;9(2):85. doi:10.4103/jmh.JMH_77_17

28. Akalanka H, Ekanayake $\overline{\mathrm{S}}$, Samarasinghe K. Could anthropometric and lipid parameters reflect susceptibility to breast Cancer? Comparison of newly diagnosed breast cancer and apparently healthy women. Asian Pac J Cancer Prev. 2018;19(9):2475.

29. Nelson ER, Chang C-Y, McDonnell DP. Cholesterol and breast cancer pathophysiology. Trends Endocrinol Metab. 2014;25 (12):649-655. doi:10.1016/j.tem.2014.10.001

30. Shah FD, Shukla SN, Shah PM, Patel HR, Patel PS. Significance of alterations in plasma lipid profile levels in breast cancer. Integr Cancer Ther. 2008;7(1):33-41. doi:10.1177/1534735407313883

31. Laamiri FZ, Otmani A, Ahid S, Barkat A. Lipid profile among moroccan overweight women and breast cancer: a case-control study. Int J Gen Med. 2013;6:439.

32. Johnson KE, Siewert KM, Klarin D, et al. The relationship between circulating lipids and breast cancer risk: a mendelian randomization study. PLoS Med. 2020;17(9):e1003302. doi:10.1371/journal

33. Kim Y, Park SK, Han W, et al. Serum high-density lipoprotein cholesterol and breast cancer risk by menopausal status, body mass index, and hormonal receptor in Korea. Cancer Epidemiol Prev Biomarkers. 2009;18(2):508-515. doi:10.1158/1055-9965.EPI-080133

34. Bade G, Shah S, Nahar P, Vaidya S. Effect of menopause on lipid profile in relation to body mass index. Chron Young Sci. 2014;5 (1):20. doi:10.4103/2229-5186.129331

35. Franssen R, Monajemi H, Stroes ES, Kastelein JJ. Obesity and dyslipidemia. Med Clin North Am. 2011;95(5):893-902. doi:10.10 16/j.mena.2011.06.003

36. Yangoua HCM, Azantsa BGK, Kuate D, et al. Characterization of dyslipidemia and assessment of atherogenic risk amongst cameroonian living in yaounde: a Cross Sectional Study. J Biosci Med. 2019;7 (7):35-50. doi:10.4236/jbm.2019.77004

37. His M, Dartois L, Fagherazzi G, et al. Associations between serum lipids and breast cancer incidence and survival in the $\mathrm{E} 3 \mathrm{~N}$ prospective cohort study. Cancer Causes Control. 2017;28(1):77-88.

38. Melvin JC, Seth D, Holmberg L, et al. Lipid profiles and risk of breast and ovarian cancer in the Swedish AMORIS study. Cancer Epidemiol Prev Biomarkers. 2012;21(8):1381-1384. doi:10.1158/ 1055-9965.EPI-12-0188

Breast Cancer: Targets and Therapy

Dovepress

\section{Publish your work in this journal}

Breast Cancer - Targets and Therapy is an international, peer-reviewed open access journal focusing on breast cancer research, identification of therapeutic targets and the optimal use of preventative and integrated treatment interventions to achieve improved outcomes, enhanced survival and quality of life for the cancer patient.

The manuscript management system is completely online and includes a very quick and fair peer-review system, which is all easy to use. Visit http://www.dovepress.com/testimonials.php to read real quotes from published authors.

Submit your manuscript here: https://www.dovepress.com/breast-cancer-targets-and-therapy-journal 